\title{
Detection of Mirai Malware Attacks in IoT Environments Using Random Forest Algorithms
}

\author{
Nur Widiyasono ${ }^{1}$, Ida Ayu Dwi Giriantari ${ }^{2}$, Made Sudarma ${ }^{2}$, L Linawati ${ }^{2}$ \\ ${ }^{1}$ Universitas Siliwangi, Siliwangi Street, Tasikmalaya 46115, Indonesia \\ ${ }^{2}$ Universitas Udayana, PB Sudirman Street, Denpasar 80234, Indonesia
}

\begin{abstract}
The potential for Cyber-attacks against Internet of Thing (IoT) Infrastructure is enormous as devices run on pre-existing network infrastructure, for example Mirai Malware Attack. Network Forensics investigations require the Random Forest Algorithm which is used to perform classification and detection techniques for the Mirai Malware attack. The trials have been carried out using 5 attack scenarios and device types. The experimental results show that the RF algorithm achieves optimal performance with an average accuracy value of $95.01 \%$, recall $90.82 \%$, F1 Score $93.85 \%$ and the best precision value $99.23 \%$. Besides, the Random Forest algorithm is suitable for very large data processing. The contribution of this research is to provide a recommendation that the $R F$ Algorithm can be used to classify and identify Mirai malware attacks on the Internet of Things infrastructure.
\end{abstract}

Keywords - IoT, Mirai Malware, Random Forest Algorithm, IOT Environment.

\section{Introduction}

The growth of various electronic devices connected to the Internet is quite good evidence of Internet of Things technology.

DOI: $10.18421 /$ TEM103-27

https://doi.org/10.18421/TEM103-27

Corresponding author: Nur Widiyasono, Universitas Siliwangi, Siliwangi Street, Tasikmalaya 46115, Indonesia.

Email: nur.widiyasono@unsil.ac.id

Received: 23 April 2021.

Revised: 08 July 2021.

Accepted: 14 July 2021.

Published: 27 August 2021.

(cc) BY-NC-ND (C) 2021 Nur Widiyasono et al; published by UIKTEN. This work is licensed under the Creative Commons Attribution-NonCommercial-NoDerivs 4.0 License.

The article is published with Open Access at www.temjournal.com
Electronic devices commonly used in households, industrial automation, and smart city infrastructure are now all connected to the Internet [1]. The security risks generated by technology development are also increasingly high. Threats to the Internet of Things (IoT) target various hardware devices, including IP cameras, home routers, and smart devices [2]. Distributed Denial of Service (DDoS) attacks are one of the most interesting types of attacks in cyberspace, where Crackers manipulates a number of devices capable of accessing the internet known as botnets and then makes simultaneous requests to servers or various servers to ensure service, so flood the server and make it ignore requests from legitimate users [3]. Malware is a malicious program [4], one of the most dangerous malware in recent years is Mirai malware, it has even been used for the biggest DDoS attacks ever recorded [5]. DDoS attacks using the Mirai botnet and launched by IoT devices tend to be large and annoying, so overcoming the Mirai botnet threat is an urgent problem. Machine learning is a solution to creating a mechanism for detecting and identifying new types of attacks, this technique provides anomalous based intrusion detection functions in IoT networks [6]. Random Forest is a machine learning classification algorithm that has been proven to perform well in conducting classification compared to other classification algorithms [7] and the random forest has the best performance for anomaly detection [8].

In this research, we used a public dataset from the UCI Repository entitled detection of IoT botnet attacks $\mathrm{N}$ BaIoT. The data tested was only a Mirai malware attack on the internet of things device which is a type of security camera. The N BaIoT dataset is collected from raw network traffic data (in pcap format) using port mirroring on the switch through which the organizational traffic typically flows [9]. Each security camera device has 6 datasets, one dataset is a type of normal traffic, five other types of attack traffic files, including Scan, ACK, SYN, UDP and UDPPlain. 
The amount of data for each dataset is very large. Therefore, in this paper, we propose machine learning methods using Random Forest (RF). This method is not only proved to be stronger [8] but also produced a high level of accuracy [10]. We also propose a sampling and features selection process to produce maximum accuracy. Sampling is used to balance the unbalanced data on a type of attack The key goal of this research is the application of random forest algorithms in detecting Mirai malware attacks, including Scan, ACK, SYN, UDP and UDPplain on the internet of things device (security cameras) and also measuring accuracy. traffic file, unbalanced data poses a big challenge for Random Forest techniques [7]. Whereas, Feature Selection (FS) is the process of selecting a subset of feature sets or attributes from a given data set while ignoring irrelevant or irrelevant features [11].

\section{Research Method}

In this study, (Fig. 1) is proposed as a research flow stages, there are 4 main stages, namely: Data Collection, Data Processing, Modeling, and Testing.

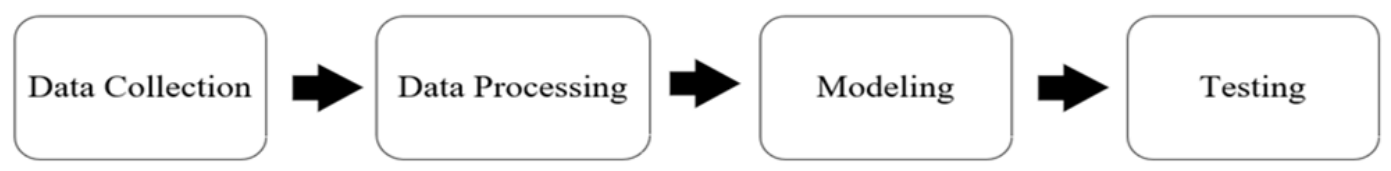

Figure 1. Research process flow

\subsection{Data Collection}

Data collection is the first stage in the research, namely the process of collecting data from various

public dataset from the UCI Repository. All information in the dataset is collected and arranged sources [12], the source of which we use is only a based on function and type. The process of collecting data in this research is described in Figure 2.

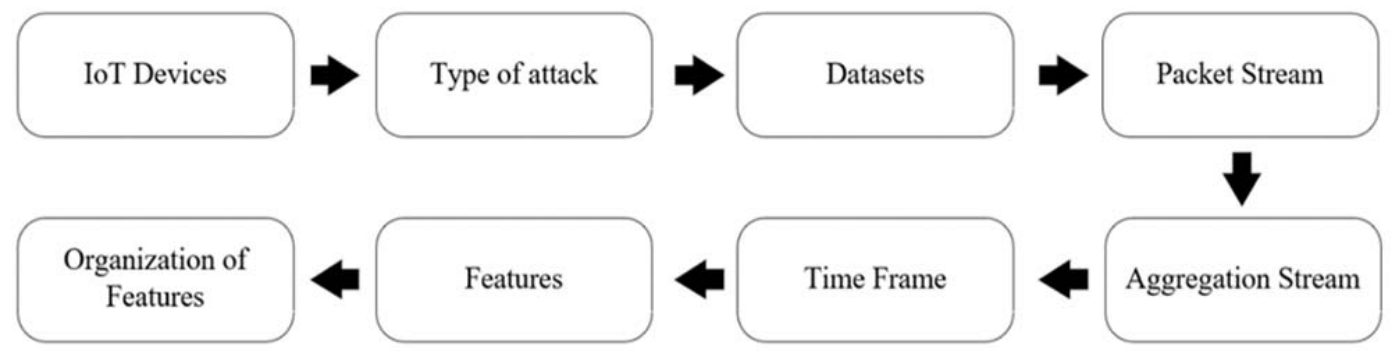

Figure 2. Data Collection Flowchart

\subsubsection{IoT Devices}

The selected IoT device is 4 IoT devices of the type of security cameras from 2 different brands. The details of the datasets such as name and type devices are shown in Table 1 and for detailed specifications such as ports and wireless support are shown in Table 2.

Table 1. IoT Devices

\begin{tabular}{cll}
\hline Code & \multicolumn{1}{c}{ Device Name } & \multicolumn{1}{c}{ Type } \\
\hline pro7 & Provision PT-737E & Security Camera \\
pro8 & Provision PT-838 & Security Camera \\
sam2 & SimpleHome XCS7-1002-WHT & Security Camera \\
sam3 & SimpleHome XCS7-1003-WHT & Security Camera \\
\hline
\end{tabular}

Table 2. IoT Device Specifications

\begin{tabular}{ccccccc}
\hline Code & Wireless Support & Port & Camera Quality & Pan/ Tilt & iOS/ Android App & 2 Way Audio \\
\hline Pro7 & $802.11 \mathrm{~b} / \mathrm{g} / \mathrm{n}$ & 80 (UDP) & 1MP (720p) & $280^{\circ} / 80^{\circ}$ & $\checkmark$ & $\checkmark$ \\
Pro8 & $802.11 \mathrm{~b} / \mathrm{g} / \mathrm{n}$ & 80 (UDP) & 2MP (1080p) & $355^{\circ} / 120^{\circ}$ & $\checkmark$ & $\checkmark$ \\
Sam2 & $802.11 \mathrm{~b} / \mathrm{g} / \mathrm{n}$ & 80 (UDP) & 1MP (720p) & $355^{\circ} / 120^{\circ}$ & $\checkmark$ & $\checkmark$ \\
Sam3 & $802.11 \mathrm{~b} / \mathrm{g} / \mathrm{n}$ & 80 (UDP) & 1MP (720p) & - & $\checkmark$ & - \\
\hline
\end{tabular}




\subsubsection{Type of Attack}

There are 5 types of Mirai malware attacks, four of the five types of attacks are Distributed Denial of
Services (DDoS) attacks. The details of the datasets are shown in Table 3.

Table 3. Type of Attack

\begin{tabular}{cl}
\hline Attacks & \multicolumn{1}{c}{ Description } \\
\hline Scan & Automatic scanning for vulnerable devices \\
ACK & Ack flooding \\
SYN & Syn flooding \\
UDP & UDP flooding \\
UDPplain & UDP flooding with fewer options, optimized \\
& for higher packets per second \\
\hline
\end{tabular}

\subsubsection{Datasets}

There are 6 datasets in each device, consisting of 1 dataset of normal traffic (Benign) and 5 datasets of
Mirai attack traffic (Scan, ACK, SYN, UDP, and UDPplain). The details are shown in Table 4.

Table 4. Datasets

\begin{tabular}{lcccccc}
\hline Code & Benign & Scan & ACK & SYN & UDP & UDPplain \\
\hline pro7 & $\checkmark$ & $\checkmark$ & $\checkmark$ & $\checkmark$ & $\checkmark$ & $\checkmark$ \\
pro8 & $\checkmark$ & $\checkmark$ & $\checkmark$ & $\checkmark$ & $\checkmark$ & $\checkmark$ \\
sam2 & $\checkmark$ & $\checkmark$ & $\checkmark$ & $\checkmark$ & $\checkmark$ & $\checkmark$ \\
sam3 & $\checkmark$ & $\checkmark$ & $\checkmark$ & $\checkmark$ & $\checkmark$ & $\checkmark$ \\
\hline
\end{tabular}

\subsubsection{Packet Stream}

There are 8 statistics extracted from the stream package, the details of the packet stream dataset are shown in Table 5.

Table 5. Packet Stream

\begin{tabular}{ll}
\hline Packet Stream & \multicolumn{1}{c}{ Description } \\
\hline Weight & The weight of the stream \\
Mean & The mean of the stream \\
Variance & The variance of the stream \\
Std & The standard deviation of the stream \\
Magnitude & The number of square roots between two streams' means \\
Radius & The number of square roots between two streams' variancles \\
Covariance & The covariance is estimated between two streams \\
Pcc & The correlation coefficient is estimated between two streams \\
\hline
\end{tabular}

\subsubsection{Agegration Stream}

Table 6 shows the details of aggregation streams. These five aggregations are the latest traffic summary statistics:

Table 6. Agregation Stream

\begin{tabular}{|c|c|c|}
\hline Code & Aggregation & Description \\
\hline MI & Host-MAC\&IP & Statistics summarizes the latest traffic from packet's host (IP + MAC) \\
\hline $\mathrm{H}$ & Host-IP & Statistics summarizes the latest traffic from packet's host (IP) \\
\hline $\mathrm{HH}$ & Channel & Statistics summarizes the latest traffic from packet's host (IP) to packet's destination host. \\
\hline $\mathrm{HH}$ _jit & Network Jitter & $\begin{array}{l}\text { Statistics summarizes the jitter of the traffic from packet's host (IP) to packet's destination } \\
\text { host. }\end{array}$ \\
\hline $\mathrm{HpHp}$ & Socket & $\begin{array}{l}\text { Statistics summarizes the latest traffic from packet's host+port (IP) to packet's destination } \\
\text { host+port. }\end{array}$ \\
\hline
\end{tabular}

\subsubsection{Time Frame}

There are 5-time frames contained in the features in the dataset, time frame 5 times is used to detect Mirai malware in real time. The details of the time frame of the dataset are shown in Table 7. 
Table 7. Time Frame

\begin{tabular}{cc}
\hline Code & Time Frame \\
\hline L5 & 1 minute \\
L3 & 10 second \\
L1 & 1.5 second \\
L0.1 & 500 millisecond \\
L0.01 & 100 millisecond \\
\hline
\end{tabular}

\subsubsection{Feature}

Table 8 shows the details of the features contained in each dataset, these features consist of 23 main

Table 8. Feature features and 5 -time frames ( 1 minute, 10 seconds, 1.5 seconds, 500 milliseconds and 100 milliseconds), the number of features in each dataset is 115 features.

\begin{tabular}{|c|c|c|c|c|c|c|c|c|c|}
\hline \multirow{2}{*}{ Code } & \multirow{2}{*}{$\begin{array}{c}\text { Time } \\
\text { Frame }\end{array}$} & \multicolumn{8}{|c|}{ Packet Stream } \\
\hline & & Weight & Mean & Variance & Std & Magnitude & Radius & Covariance & Pcc \\
\hline \multirow{5}{*}{ MII } & L5 & $\checkmark$ & $\checkmark$ & $\checkmark$ & & & & & \\
\hline & L3 & $\checkmark$ & $\checkmark$ & $\checkmark$ & & & & & \\
\hline & L1 & $\checkmark$ & 2 & $\checkmark$ & & & & & \\
\hline & L0.1 & $\checkmark$ & $\checkmark$ & $\checkmark$ & & & & & \\
\hline & L0.01 & $\checkmark$ & 2 & $\checkmark$ & & & & & \\
\hline \multirow{5}{*}{ H } & L5 & $\checkmark$ & $\checkmark$ & $\checkmark$ & & & & & \\
\hline & L3 & $\checkmark$ & 2 & $\checkmark$ & & & & & \\
\hline & L1 & $\checkmark$ & $\checkmark$ & $\checkmark$ & & & & & \\
\hline & L0.1 & $\checkmark$ & $\checkmark$ & $\checkmark$ & & & & & \\
\hline & L0.01 & $\checkmark$ & $\checkmark$ & $\checkmark$ & & & & & \\
\hline \multirow{5}{*}{$\mathrm{HH}_{j}$ jit } & L5 & $\checkmark$ & $\checkmark$ & $\checkmark$ & & & & & \\
\hline & L3 & $\checkmark$ & $\checkmark$ & $\checkmark$ & & & & & \\
\hline & L1 & $\checkmark$ & 2 & $\checkmark$ & & & & & \\
\hline & L0.1 & $\checkmark$ & $\checkmark$ & $\checkmark$ & & & & & \\
\hline & L0.01 & $\checkmark$ & 2 & $\checkmark$ & & & & & \\
\hline \multirow{5}{*}{ HH } & L5 & $\checkmark$ & 2 & & $\checkmark$ & $\checkmark$ & $\checkmark$ & $\checkmark$ & $\checkmark$ \\
\hline & L3 & $\checkmark$ & 2 & & $\checkmark$ & $\checkmark$ & $\checkmark$ & $\checkmark$ & $\checkmark$ \\
\hline & L1 & $\checkmark$ & 2 & & $\checkmark$ & $\checkmark$ & $\checkmark$ & 2 & $\checkmark$ \\
\hline & L0.1 & 2 & $\checkmark$ & & $\checkmark$ & $\checkmark$ & $\checkmark$ & $\checkmark$ & $\checkmark$ \\
\hline & L0.01 & 2 & 2 & & $\checkmark$ & $\checkmark$ & $\checkmark$ & 2 & $\checkmark$ \\
\hline \multirow{5}{*}{ НрНр } & L5 & $\checkmark$ & $\checkmark$ & & $\checkmark$ & $\checkmark$ & $\checkmark$ & $\checkmark$ & $\checkmark$ \\
\hline & L3 & $\checkmark$ & $\checkmark$ & & $\checkmark$ & $\checkmark$ & $\checkmark$ & 2 & $\checkmark$ \\
\hline & L1 & $\checkmark$ & $\checkmark$ & & $\checkmark$ & $\checkmark$ & $\checkmark$ & $\checkmark$ & $\checkmark$ \\
\hline & L0.1 & $\checkmark$ & 2 & & $\checkmark$ & $\checkmark$ & $\checkmark$ & $\checkmark$ & $\checkmark$ \\
\hline & L0.01 & $\checkmark$ & 2 & & $\checkmark$ & $\checkmark$ & $\checkmark$ & 2 & 2 \\
\hline
\end{tabular}

\subsubsection{Organization of Features}

Table 9 shows the organization of feature datasets based on package statistics in Table 8 . This Feature Organization has 4 groups.

Table 9. Organization of Features

\begin{tabular}{ccccc}
\hline \multirow{2}{*}{$\begin{array}{c}\text { Aggregate } \\
\text { (packet src, dst) }\end{array}$} & \multicolumn{4}{c}{ Statistic Packet with Matrix } \\
\cline { 2 - 5 } & Count & Jitter & Outbound Size & Combined Size \\
\hline $\mathrm{MI}$ & weight & & mean, variance & \\
$\mathrm{H}$ & weight & & mean, variance & \\
$\mathrm{HH}$ & weight & weight, mean, variance & mean, variance & magnitude, radius, covariance, pcc \\
$\mathrm{HpHp}$ & weight & & mean, variance & magnitude, radius, covariance, pcc \\
\hline
\end{tabular}

\subsection{Data Processing}

Data processing is the second stage in research, be used for the testing process. Data processing in namely processing datasets with large amounts of this research is described in Figure 3. data and unbalanced data, becoming datasets that can 


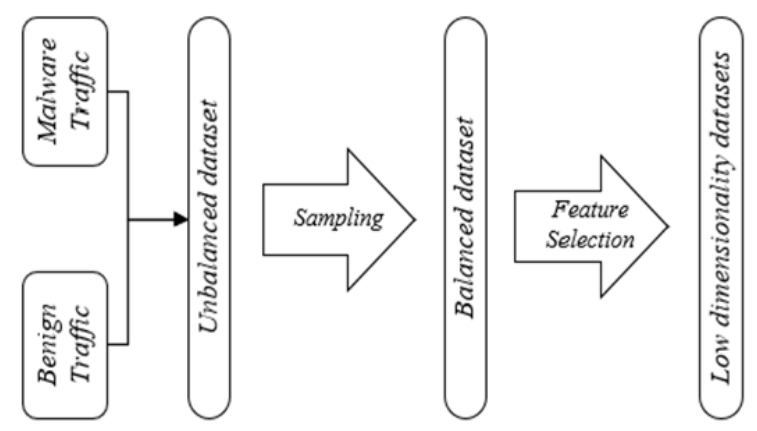

Figure 3. Data Processing Flowchart

Figure 3 shows the stages of data processing, the beginning of the process of entering a dataset of normal traffic and attack traffic, and the combination produces an unbalanced dataset, then the dataset is sampled so that the combined dataset of traffic and attack traffic becomes a balanced dataset. However, the dimensionality dataset is still high, then the feature in the dataset is selected so that the dataset becomes low dimensionality and the accuracy level becomes optimal.

\subsubsection{Sampling}

Sampling is the process of randomly selecting data to make subsets. This sampling aims to cut a lot of data into a little, a little data processing process results in a faster machine learning process. Random sampling is a sampling method that uses calculations based on "random numbers" [13]. The formula used for sampling can be seen in equation (1) below:

$$
\frac{\text { (Total number of examples) }}{\text { (sum of ratios }} \times \text { ratio of this partition }
$$

\subsubsection{Feature Selection}

Features Selection aims to select the most important features for a random forest in the classification of benign traffic and malicious traffic. Classification is used to map input data to predetermined class labels [14]. Features selection selects the 5 best features of 115 features. Random Forest is used as a selection process based on Tree Importance from 100 decision trees with a depth of 10 , the processing criteria used by random forest is the Gini index.

\subsection{Modeling}

Modeling is the third stage in research. Modeling is the process of designing a model for feature selection (Figure 4), testing and anomaly detection (Figure 5). Process flow stages of research can be described in Figure 1.

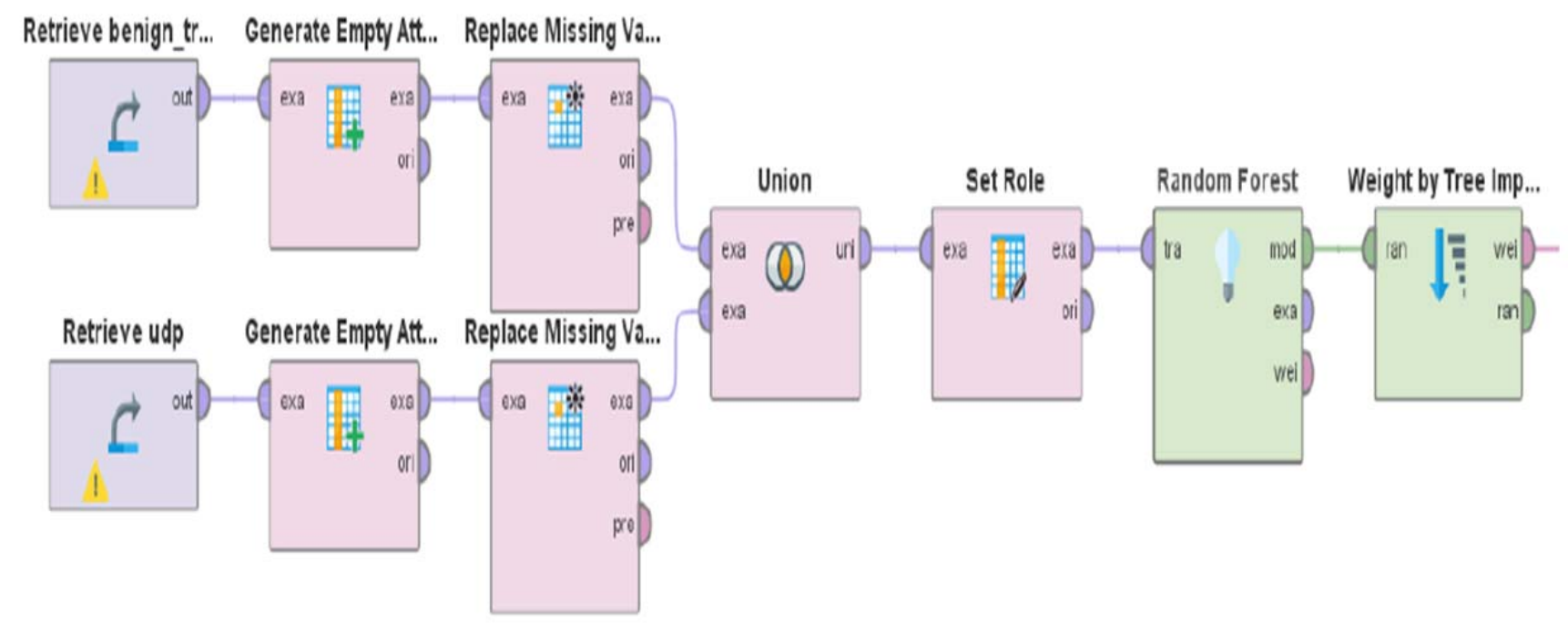

Figure 4. Feature Selection Model

The modeling in Figure 4 shows how to select features. The dataset used is a dataset with the highest amount of data in normal traffic (Benign) and attack traffic (UDP) on PT-838 Provision devices. This model does not use a sampling dataset, a combination of normal traffic and attack traffic is then processed using a random forest algorithm to find the best feature accuracy based on the Gini index criteria using the Tree Importance decision. 


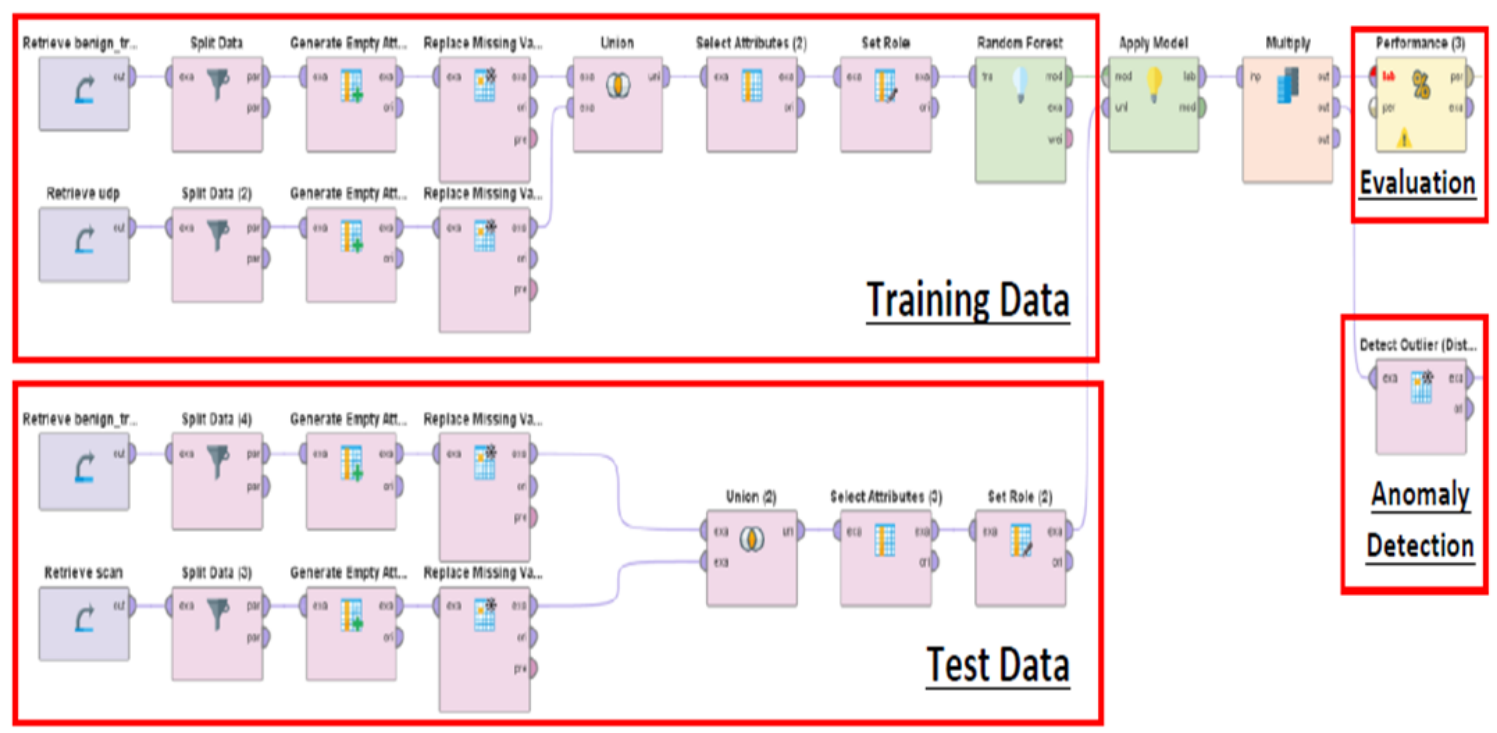

Figure 5. Testing Model

The modeling in Figure 5 shows how the testing process starts by selecting the normal traffic dataset and attack traffic in the training data and test data. Training data is the process of modeling the algorithm used to be studied by the system, while the test data is the modeling process that the system has learned through the training phase and it will be used to produce a certain decision. The amount of data in the training data and test data is balanced first using sampling. Then, we choose the 5 best features to get optimal results. Then, the classification of test data based on training data will be processed. In the evaluation process model, only the performance operator is required to calculate accuracy, recall, precision, and f-measure. The anomaly detection model is also the same, only adding 1 operator is to detect outlier (Distanced).

\section{Results and Discussion}

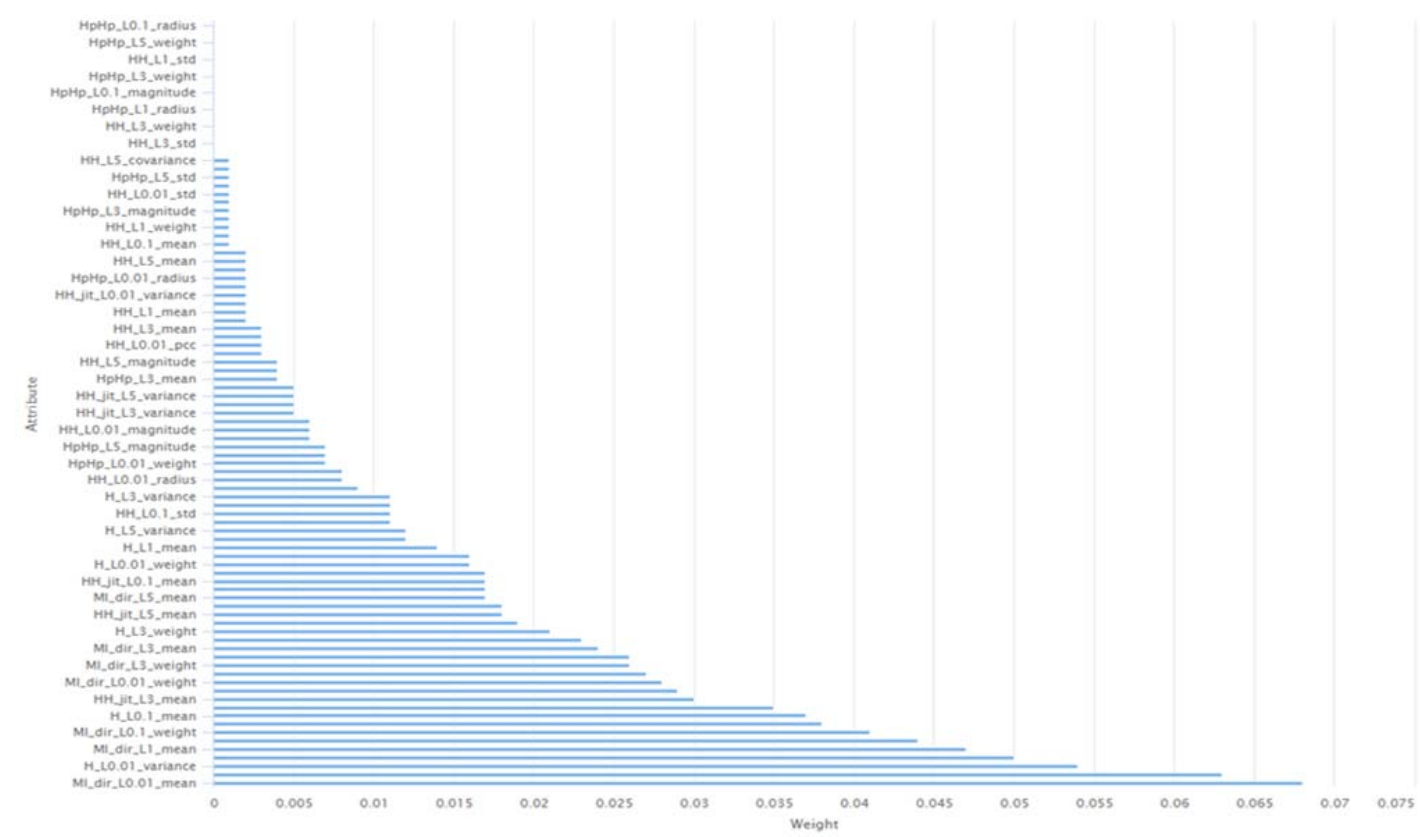

Figure 6. Gini Index Accuracy (Increased) Random Forest Model

Figure 6, shows the results of the Gini Index Accuracy (Increase) using Tree Importance with the
Random Forest Model, five features with the highest accuracy of the Gini index is shown in Table 10. 
Table 10. The Best Feature

\begin{tabular}{l}
\hline \multicolumn{1}{c}{ Features } \\
\hline Host-MAC\&IP_100ms_Mean \\
Host-MAC\&IP_500ms_Mean \\
Host-IP_500ms_Variance \\
Host-MAC\&IP_1,5s_Weight \\
Host-MAC\&IP_1,5s_Mean \\
\hline
\end{tabular}

The process of testing algorithms is done using the confusion matrix, the method is shown in Table 11 below:

Table 11. Confusion Matrix

\begin{tabular}{|c|c|c|}
\hline Prediction Actual & Attack & Normal \\
\hline Attack & TP & FN \\
\hline Normal & FP & TN \\
\hline
\end{tabular}

Positive-Positive (TP) is the amount of attack traffic that is predicted as attack traffic, FalsePositive (FP) which is the amount of attack traffic predicted as normal, False-Negative (FN) traffic which is the amount of normal traffic predicted as attack traffic and True-Negative (TN), which is the with equations (2), (3), (4) and (5).

$$
\begin{array}{ll}
\text { Accuracy } & =\frac{\mathrm{TP}+\mathrm{TN}}{\mathrm{TP}+\mathrm{TN}+\mathrm{FP}+\mathrm{FN}} \\
\text { Recall } & =\frac{\mathrm{TP}}{\mathrm{TP}+\mathrm{FN}} \sigma \\
\text { Precision } & =\frac{\mathrm{TP}}{\mathrm{TP}+\mathrm{FP}} \\
\mathrm{F}-\text { Measure } & =2 \times \frac{\text { Precision } \times \text { Recall }}{\text { Precision }+ \text { Recall }}
\end{array}
$$

\section{Testing I}

Testing I, tests normal network traffic (benign) and attack network traffic (Scan) on the PT-737E amount of normal traffic predicted as normal traffic. The results of the confusion matrix are used to measure accuracy, precision, recall, and Fmeasure to analyze the performance of algorithms in classifying to detect Mirai malware

Table 12. Confusion Matrix Testing I

\begin{tabular}{|c|c|c|}
\hline Actual & Attack & Normal \\
\hline Attack & 573 & 0 \\
\hline Normal & 427 & 1000 \\
\hline
\end{tabular}

Based on confusion matrix testing 1, the data of accuracy, recall, precision and F-measure are obtained, as follows:

Provision device. The confusion matrix of the test results is summarized in Table 12.

$$
\begin{array}{ll}
\text { Accuracy } & =\frac{573+1000}{573+1000+427+0}=78,65 \% \\
\text { Recall } & =\frac{573}{573+427}=57,30 \% \\
\text { Precision } & =\frac{573}{573+0}=100 \% \\
\text { F-Measure } & =2 \times \frac{100 \times 57,30}{100+57,30}=72,85 \%
\end{array}
$$




\section{Testing II}

Testing II, tests normal network traffic (benign) and 838 device. The confusion matrix of the test results is attack network traffic (ACK) on the Provision PT- summarized in Table 13.

Table 13. Confusion Matrix Testing II

\begin{tabular}{|c|c|c|}
\hline Actual & Attack & Normal \\
\hline Prediction & 973 & 0 \\
\hline Normal & 27 & 1000 \\
\hline
\end{tabular}

Based on confusion matrix testing II, the data of accuracy, recall, precision and F-measure are obtained, as follows:

$$
\begin{array}{ll}
\text { Accuracy } & =\frac{973+1000}{973+1000+27+0}=98,65 \% \\
\text { Recall } & =\frac{973}{973+27}=97,30 \% \\
\text { Precision } & =\frac{973}{973+0}=100 \% \\
\text { F-Measure } & =2 \times \frac{100 \times 97,30}{100+97,30}=98,63 \%
\end{array}
$$

\section{Testing III}

Testing III, tests normal network traffic (benign) XCS7-1002-WHT device. The confusion matrix of and attack network traffic (SYN) on the SimpleHome the test results is summarized in Table 14.

Table 14. Confusion Matrix Testing III

\begin{tabular}{|c|c|c|}
\hline Actual & Attack & Normal \\
\hline Attack & 995 & 0 \\
\hline Normal & 5 & 1000 \\
\hline
\end{tabular}

Based on confusion matrix testing III, the data of accuracy, recall, precision and F-measure are obtained, as follows:

$$
\begin{array}{ll}
\text { Accuracy } & =\frac{1000+960}{1000+960+40+0}=98,00 \% \\
\text { Recall } & =\frac{1000}{1000+0}=100 \% \\
\text { Precision } & =\frac{1000}{1000+40}=96,15 \% \\
\text { F-Measure } & =2 \times \frac{96,15 \times 100}{96,15+100}=98,04 \%
\end{array}
$$

\section{Testing IV}

Testing IV, tests normal network traffic (benign) XCS7-1003-WHT device. The confusion matrix of and attack network traffic (UDP) on the SimpleHome the test results is summarized in Table 15.

Table 15. Confusion Matrix Testing IV

\begin{tabular}{|c|c|c|}
\hline Prediction Actual & Attack & Normal \\
\hline Attack & 1000 & 0 \\
\hline Normal & 0 & 1000 \\
\hline
\end{tabular}

Based on confusion matrix testing IV, the data of accuracy, recall, precision and F-measure are obtained, as follow: 


$\begin{array}{ll}\text { Accuracy } & =\frac{1000+1000}{1000+1000+0+0}=100 \% \\ \text { Recall } & =\frac{1000}{1000+0}=100 \% \\ \text { Precision } & =\frac{1000}{1000+0}=100 \% \\ \text { F-Measure } & =2 \times \frac{100 \times 100}{100+100}=100 \%\end{array}$

\section{Testing $V$}

Testing V, tests normal network traffic (benign) Provision PT-737E device. The confusion matrix of and attack network traffic (UDPplain) on the the test results is summarized in Table 16.

Table 16. Confusion Matrix Testing V

\begin{tabular}{|c|c|c|}
\hline Prediction Actual & Attack & Normal \\
\hline Attack & 1000 & 0 \\
\hline Normal & 40 & 960 \\
\hline
\end{tabular}

Based on confusion matrix testing $\mathrm{V}$, the data of accuracy, recall, precision and F-measure are obtained, as follows:

$\begin{array}{ll}\text { Accuracy } & =\frac{995+1000}{995+1000+5+0}=99,75 \% \\ \text { Recall } & =\frac{995}{995+5}=99,50 \% \\ \text { Precision } & =\frac{995}{995+0}=100 \% \\ \text { F-Measure } & =2 \times \frac{100 \times 99,50}{100+99,50}=99,75 \%\end{array}$

Tests 1 to 5 produce statistical data to be evaluated, statistical data is shown in Table 17 and graph in Figure 6.

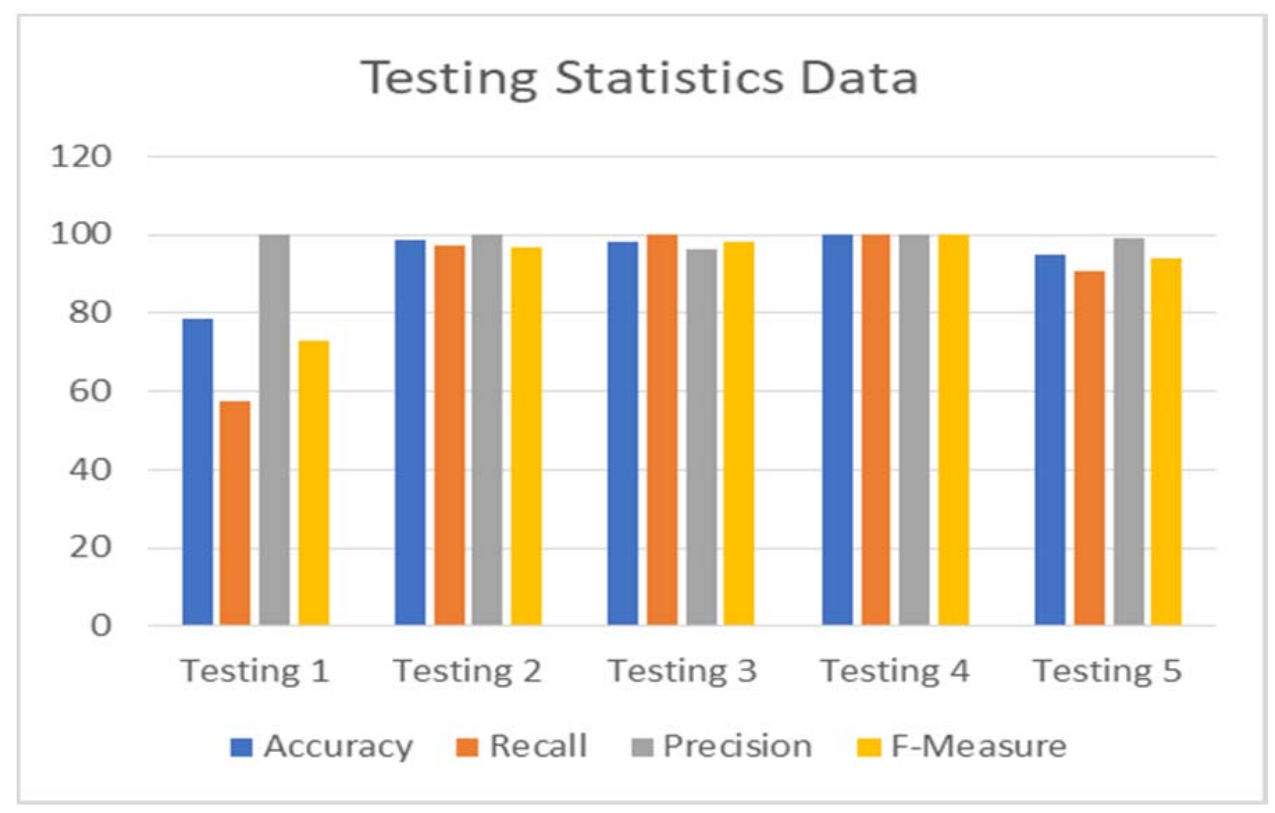

Figure 7. Testing Statistics Data

Figure 7 shows that testing 1 produces different statistical data compared to the other 4 testings. This is because training data is used for cross attack networks, using the UDP dataset. The type of UDP attack is the same as the type of ACK, SYN and UDPplain attacks, which is the type of attack that floods the Internet of Things or into the Distributed Denial of Services (DDoS) type of attack. The test 
data on testing 1 uses the Scan attack dataset, which is an attack by scanning vulnerable Internet of Things devices. Thus, the statistical data on testing 1 produces accuracy, recall, and measure which are lower than the results of another testing.

\section{Anomaly Detection}

Normal traffic and attack traffic, previously processed first from training data and test data using the random forest, as in the testing process 1 to 5 , then the output is entered into detect outlier (Distanced). Scatter 3D for normal traffic and attack traffic is shown in Figure 7, while Scatter 3D outliers are in Figure 8.

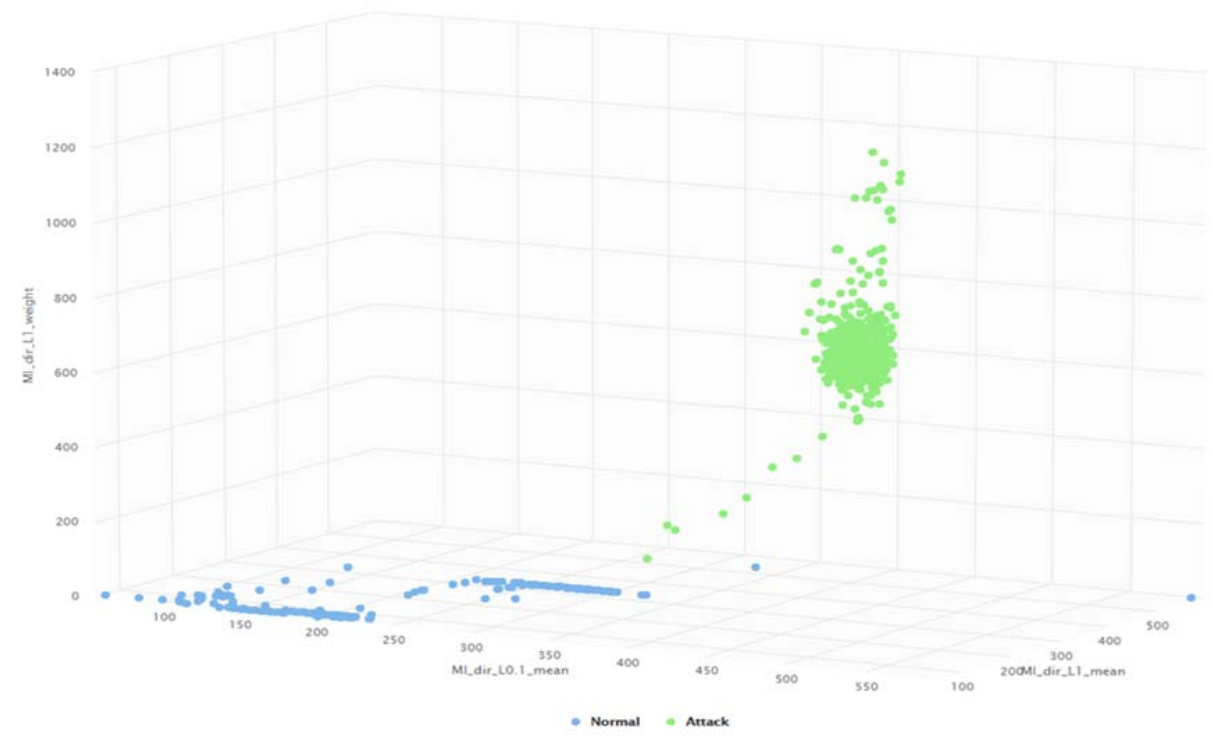

Figure 8. Scatter 3D Normal Traffic (blue dots) Attack Traffic (green dots)

Scatter 3D in Figure 8 shows normal traffic (blue dot) and attack traffic (green dot). 3D Scatter consists of 3 features, on the $\mathrm{x}$-axis is Host-MAC \& IP $500 \mathrm{~ms}$ Mean, on the y-axis, there is the Host-MAC \& IP $500 \mathrm{~ms}$ Mean and finally, the z-axis is Host-MAC \&
IP $5 \mathrm{~s}$ Weight. The three features are selected from the 5 best sequences from the feature selection stage (Table 10). The 3D Scatter color is then changed and based on the type of outlier or anomaly.

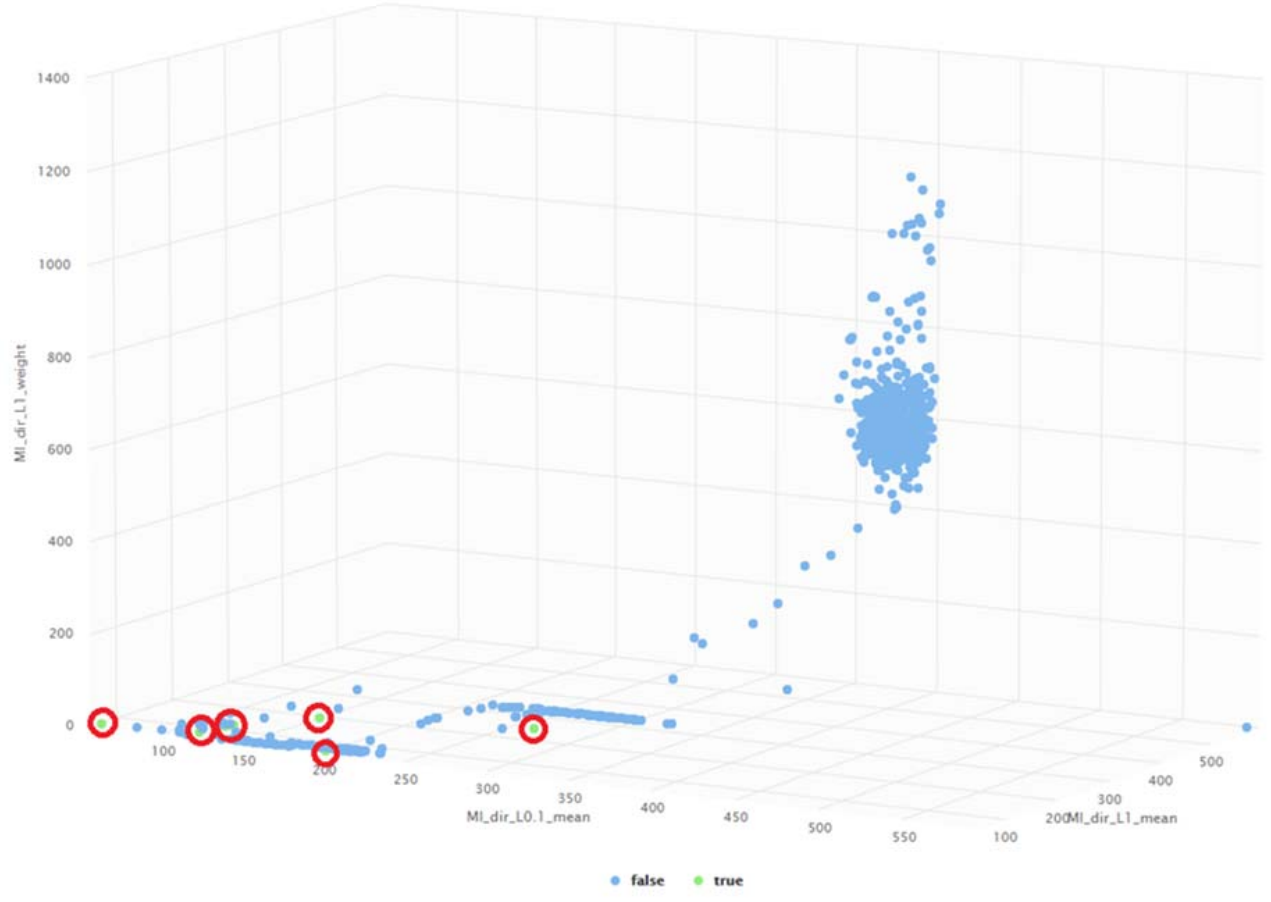

Figure 9. Scatter 3D Outlier False (blue dots) Outlier True (green dots) 
Figure 9 shows some outliers (green dots) in normal traffic (Figure 8). Green dots are detected as outliers or anomalies found in the Host-MAC \& IP $500 \mathrm{~ms}$ Mean and Host-MAC \& IP $500 \mathrm{~ms}$ features. Detect outlier (Distanced) is used for anomaly detection because the output is in the form of boolean (true and false), so it can be directly visualized through Scatter 3D.

\section{Conclusion}

An algorithm called Random Forest (RF) has been proposed to detect Mirai malware. The proposed technique produces an average accuracy value of $95.01 \%$ and produces an average precision value of $99.23 \%$. Moreover, the selection of the right features also helps us produce high detection accuracy. Regarding testing for anomaly detection using the detect outlier (Distanced) technique, it successfully detected outliers/ anomalies in the Host-MAC \& IP 500ms_Mean and Host-MAC \& IP 500ms features.

\section{References}

[1]. Bastos, D., Shackleton, M., \& El-Moussa, F. (2018, March). Internet of Things: A survey of technologies and security risks in smart home and city environments. In Living in the Internet of Things: Cybersecurity of the IoT-2018 (pp. 1-7). IET.

[2]. C. Boom, T. Continue, and T. A., (2019). Motivated, "McAfee Labs Threats Report: December 2018," Comput. Fraud Secur., 2019(1), p. 4.

[3]. Angrishi, K. (2017). Turning internet of things (iot) into internet of vulnerabilities (iov): Iot botnets. arXiv preprint arXiv:1702.03681.

[4]. Aldya, A. P., Widiyasono, N., \& Setia, T. P. (2019). Reverse Engineering untuk Analisis Malware Remote Access Trojan. J. Edukasi dan Penelit. Inform, 5(1), 40.

[5]. De Donno, M., Dragoni, N., Giaretta, A., \& Spognardi, A. (2018). DDoS-capable IoT malwares: Comparative analysis and Mirai investigation. Security and Communication Networks.

[6]. Nõmm, S., \& Bahşi, H. (2018, December). Unsupervised anomaly based botnet detection in IoT networks. In 2018 17th IEEE international conference on machine learning and applications (ICMLA) (pp. 1048-1053). IEEE.

[7]. Agusta, Z. P., \& Adiwijaya, A. (2019). Modified balanced random forest for improving imbalanced data prediction. International Journal of Advances in Intelligent Informatics, 5(1), 58-65.

[8]. Abraham, B., Mandya, A., Bapat, R., Alali, F., Brown, D. E., \& Veeraraghavan, M. (2018, July). A comparison of machine learning approaches to detect botnet traffic. In 2018 International Joint Conference on Neural Networks (IJCNN) (pp. 1-8). IEEE.

[9]. Meidan, Y., Bohadana, M., Mathov, Y., Mirsky, Y., Shabtai, A., Breitenbacher, D., \& Elovici, Y. (2018). N-baiot-network-based detection of iot botnet attacks using deep autoencoders. IEEE Pervasive Computing, 17(3), 12-22.

[10]. Mathur, L., Raheja, M., \& Ahlawat, P. (2018). Botnet detection via mining of network traffic flow. Procedia computer science, 132, 1668-1677.

[11]. Usman, A. M., Yusof, U. K., \& Naim, S. (2018). Cuckoo inspired algorithms for feature selection in heart disease prediction. International Journal of Advances in Intelligent Informatics, 4(2), 95-106.

[12]. Muhammad, A. H. (2017). Metode Klasifikasi dan Analisis Karakteristik Malware Menggunakan Konsep Ontologi (Doctoral dissertation, Universitas Islam Indonesia).

[13]. Xie, H., Ahmadon, M. A. B., Yamaguchi, S., \& Toyoshima, I. (2019, January). Random Sampling and Inductive Ability Evaluation of Word Embedding in Medical Literature. In 2019 IEEE International Conference on Consumer Electronics (ICCE) (pp. 14). Ieee.

[14]. Ismi, D. P., Panchoo, S., \& Murinto, M. (2016). Kmeans clustering based filter feature selection on high dimensional data. International Journal of Advances in Intelligent Informatics, 2(1), 38-45. 